\title{
3-D Discrimination of Buried Object in Subsurface Soil via Magnetic Sensors
}

\author{
Daniel C. Chin, R. Srinivasan, and Robert E. Ball \\ Johns Hopkins University Applied Physics Laboratory, Johns Hopkins RD., Laurel, MD 20723 \\ E-mail: daniel.chin@ibuapl.edu
}

\begin{abstract}
The Electrical Conductivity Object Locator (ECOL) has been developed with the goal of detecting buried objects. Its specific capability to detect and characterize small-size plastic and metal objects buried at shallow depths is demonstrated. The technique can also detect larger objects at greater depths. The ECOL technique maps the soil subsurface conductivity and identifies variations in the conductivity between buried objects and their surroundings. The subsurface conductivity is mapped in two major steps: 1) Low-frequency $(1$ to $100 \mathrm{~Hz})$ and low-amplitude $(<200$ $\mu \mathrm{A})$ currents injected into the soil induce potential and magnetic fields in and around the subsurface soil. The potential and magnetic fields are measured using appropriate sensors placed on or above the soil surface. 2) Using the measured values as boundary conditions, a fast optimization algorithm, and an accurate matrix inversion routine, the subsurface conductivity is estimated. Two field tests are conducted using magnetic sensor in either contact or non-contact technique. Both tests successfully located the buried plastic and metal objects within a radius of $1.2 \mathrm{ft}$.
\end{abstract}

KEYWORD: Inversion process, Simultaneous Perturbation Stochastic Approximation, Finite Element Method, De-mine

\section{Introduction}

The Electrical Conductivity Object Locator (ECOL) uses electric conductivity maps to distinguish buried foreign objects from regular subsurface soil. Assuming that the foreign objects and the regular soil have different electrical conductivities, when an electrical current is induced into the subsurface, the difference in conductance causes distortions in the electromagnetic field. Theoretically, one can measure the distortion outside of the field to solve the subsurface conductivity profile. The challenges are the non-homogeneous subsurface modeling problem, the nonlinear effect of field distortion from the foreign object, illconditioned transponder matrix, non-uniform of the soil contents, and the multiple representation problems.

ECOL applies a low-amplitude $(\sim 200-\mu \mathrm{A})$ electric alternating current, single or multiple frequencies. The impressed $\mathrm{AC}$ current generates $A C$ potentials and magnetic field throughout the site, which are measured at the surface and the boundary of the site. ECOL utilizes the finite element method (FEM) to compute the surface and boundary potential values from the amount of current and an assumed or previously estimated conductivity profile for the subsurface. ECOL iteratively estimates the conductivity profile of the subsurface and the location and size of the buried object by minimizing the sum of the square of the differences between the measured and the computed potentials or magnetic field readings at the boundary. The minimization is based on a gradient approximation technique, namely, simultaneous perturbation stochastic approximation (SPSA) [1,2]. Chin [3] has shown that the modified SPSA could find the global minimum for multiple representation problems.

The substance in the subsurface directly affects the electric current flow. When algorithms in [4-8] are used in an environment like human body, the biological substance and structure of which are well defined, then the algorithms can extrapolate the electric potential and current strength from the surface of the object to the area of interest to compute the object conductivity. However, the soil subsurface has a vast variety of different substance and is mostly non-uniform. ECOL has to relay an inversion technique to estimate the subsurface conductivity profile for the entire subsurface. The available measuring space usually has lower dimension than the dimension of the conductivity profile space. This often creates a multiplerepresentation problem.

ECOL divides the subsurface in two areas, the area for searching the object and the area outside of the search area that affects current flow. The model for the outside search area, background environmental area, will be estimated using large size FEM elements. Each FEM element represents a mixed substance rectangular block. The search area, the area of interest, has to be detailed enough to distinguish the elements of the FEM model that includes part of the foreign 
object and the elements that do not include the foreign object. Therefore, the area of interest will be modeled with equal-size small elements. The division is necessary for saving the computation time and for reducing the ambiguity possibility of the subsurface FEM model representation.

The FEM element and the foreign object do not necessarily match in size. The size of the FEM element could be $3-4$ times larger than the foreign object. The estimates of the conductivity values for the FEM elements are the exponential weighted average of the conductivity in each element. If the element is a mostly foreign object the estimated conductivity will be near the conductivity value of the foreign object. If the foreign object occupies a very small portion of the FEM element, the conductivity estimates will be near the soil conductivity value. Then, the existence of the foreign object may be overlooked. In other words, ECOL is not sensitive to a foreign substance that is relatively small portion of the element in size, such as small pebbles, roots, or the tab from a soda-can.

To reduce the non-linearity effect due to the high contrast in the conductivity values between the foreign object and the regular soil, ECOL uses exponential units in the perturbation and estimation process. The computation in FEM is still using the regular conductivity unit. High precession inversion algorithm was implemented to handle the ill-conditioned transponder matrix in the FEM calculations. The matrix is converted to a unitary matrix before inverting. Mixing a part of the foreign object with regular soil substance in each FEM element has also helped the condition of the transponder matrix.

There are two types of measurements used for sensing the electromagnetic field disturbance: the differential-potential and magnetic field reading. The differential-potentials measure the disturbance in the surface current along the direction of input/output poles connecting the power source. The amount and the disturbance of the current flow on the top of the subsurface could reflect the substance contained beneath the surface. A simple two-dimensional FEM model could be used for discriminating the foreign object happens to be located direct under or nearby the plane defined by the two electric induction poles. The 2-D model models the plane perpendicular to the surface. The field study in Chin, et al. [8] has shown that ECOL located the mine and identified its size in the subsurface using the differential-potentials with 2-D FEM model.

The magnetic field measurement is sensitive to all currents in and around the subsurface. The magnetic field reading senses the presence of the foreign object from the current in the foreign object directly. If there are enough magnetic field measurements, one can estimate a detailed 3-D conductivity profile to separate the foreign object from the regular soil. The field readings are also sensitive to the details in the environment, mainly the wires that induce the currents to and from the subsurface. In order to have accuracy in estimation, the detailed environmental adjustment model is required in processing the magnetic field measurements. However, magnetic field measurements are more difficult to process than the potential measurements. Magnetic field measurements are available in most situations. Also, the magnetic field reading senses the currents in the object directly whereas the potential measurements are indirect. The magnetic field measurement would have stronger information for estimating the conductivity profile and identifying the foreign object.

This paper discusses the magnetic field sensor implementation, 3-D FEM model, and field demonstrations using the magnetic field readings in both contact and non-contact methods.

\section{3-Dimensional Finite Element Model}

The FEM method is based on generalized Laplace equation. For a 3-D model, this equation is of the form:

$$
\nabla(\sigma \nabla \phi)=-\frac{\rho}{\varepsilon_{0}}
$$

or

$$
\frac{\partial}{\partial x}\left(\sigma \frac{\partial \phi}{\partial x}\right)+\frac{\partial}{\partial y}\left(\sigma \frac{\partial \phi}{\partial y}\right)+\frac{\partial}{\partial z}\left(\sigma \frac{\partial \phi}{\partial z}\right)=-\frac{\rho}{\varepsilon_{0}}
$$

where $\sigma$ is the conductivity, $\phi$ is the internal voltage, $\rho$ is the internal charge density and is non-zero only in the areas that contain a current source, and $\varepsilon_{0}$ is the free space 
permittivity. In FEM, the subsurface or object under consideration is divided into different regions, each one representing one conductivity value. Each region is subdivided into small elements with constant conductivity $\left(\sigma_{I}\right.$, where the subscript $i$ represents the $i^{\text {th }}$ element conductivity), and when put together, all the regions within the subsurface are represented by a piece-wise homogeneous model.

The electrical current is defined at every location at or within the boundaries of the subsurface using the Neumann conditions. The FEM method is then used to compute the electrical potential everywhere within the subsurface. The mathematical description of the Neumann boundary conditions is described as follows:

For a body surrounded by air, the active current electrodes, with current density $J$, has:

$$
-\sigma \frac{\partial \phi}{\partial n}=J
$$

on the electrodes; and

$$
-\sigma \frac{\partial \phi}{\partial n}=0
$$

at the object-air interface, where $\partial \phi / \partial n$ is the derivative of the potential normal to the surface. The air (of the object-air interface) represents a phase that is not of interest to our study, but is present adjacent to the object under investigation.

The solution for Equations (1) and (2) is found to be equivalent to the minimization of the following functional: $F(\phi)=\frac{1}{2} \iint_{s}\left\{\sigma\left(\frac{\partial \phi}{\partial x}\right)^{2}+\sigma\left(\frac{\partial \phi}{\partial y}\right)^{2}+\sigma\left(\frac{\partial \phi}{\partial z}\right)^{2}\right\} d x d y d z+\int_{s} \frac{\rho}{\varepsilon} \phi d n$ This is called the Galerkin's error minimization method [10]. For minimizing $F(\phi)$, its partial derivatives with respect to the nodal voltages of each element must be zero. Hence, the equation

$$
\frac{\partial F(\phi)}{\partial \phi}=0
$$

holds for every node (the sides of the element). Note that the elements can have a wide variety of shapes and sizes, and each element will be associated with as many numbers of nodes as the number of sides (or corners) that it has. There is no analytical solution for (6). An iterative global optimization technique [3] based on SPSA is used in ECOL.

\section{The Iterative Global Optimization Algorithm}

The ECOL technique maps and creates images of the conductivity profiles of the subsurface at the region of interest (the rectangular grid area in Fig. 1) by injecting electrical currents into the soil. It could be a noninvasive technique that works in four major steps (see Fig. 2). Step 1 provides the experimental data needed for the reconstruction procedure described in Steps 2 through 4 that locates and characterizes the buried object.

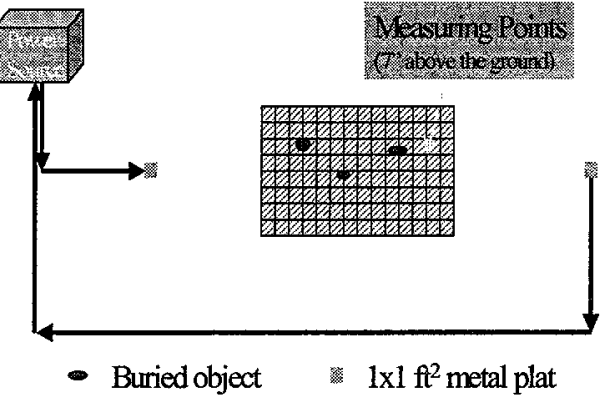

Figure 1: The Top View of the $2^{\text {nd }}$ Test Site

Step 1: Two electrodes are placed at center of opposite ends away from the test site and connected to a power source (see Fig. 1). An electric current is injected into the site. The electric current generates in electromagnetic field for the entire site, including the surface.

Step 2: The electromagnetic field generated in Step 1 is measured using the sensor shown in the picture above Block 1 in Fig. 2. The field measurements were taken at the white marks, 7" above the grid points in Fig. 2. The measurements will be adjusted by the magnetic field values generated by the current flow from the power source to induction plates (the square marks in Fig. 1).

Step 3: The subsurface of the site is divided into many elements according to the interested area and the background environmental area (Block 2 in Fig. 2). An example of the element size is shown in Fig. 4. Each element is given an initial arbitrary conductivity value (Block 3 in Fig. 2).

Step 4: This step is an iterative step. Each iteration (Block 4 in Fig. 2) consists of 3 units: SPSA estimation, FEM conversion, and ECOL evaluation.

a) SPSA estimation constructs a perturbation array from a random set of numbers generated in Bernoulli distribution with outcome \pm 1 . The perturbation array is used for perturbing the initial subsurface conductivities or the values estimated in the previous iteration. The perturbations were controlled by a step gain constant that was governed by SPSA rules as shown in Spall [1] and Chin [3]. Two perturbed conductivity profiles were made from adding and subtracting the perturbations.

b) FEM computes the voltage potentials for every element within the soil subsurface from the perturbed conductivity profiles with the Neumann boundary conditions.

c) ECOL evaluation is to compare the magnetic field measurements against the magnetic field strengths integrated from the voltage potentials at every node. 
The magnetic force and current relationship is stated at Equation 5.35 on page 105 in [11] as follows:

$$
H=\frac{I \delta l}{4 \pi r^{2}} \sin \theta
$$

d) The total square error sums are used for the objective values in SPSA. SPSA uses the evaluation errors to form an approximation gradient that updates the conductivity profile as the new estimates of this iteration.

e) A specified number ends this iterative step.

\section{Reconstruction of the Internal Conductivity of the Mine Location}

This section presents a brief description of the procedure that generates the soil subsurface conductivity map. A formal description of two important aspects of the reconstruction process was described in Step 4, SPSA and FEM in Iterative Global Optimization Algorithm section. A complete discussion of FEM can be found in [10]. The reconstruction process solves the generalized Laplace equations with Neumann boundary conditions. This equation is called the Galerkin's error minimization method. A 2-D FEM model of the experimental site for computing the potentials is shown in Fig. 3 that was reported in Chin, et al. [9]. It represents a cross section of the subsurface $100 \mathrm{~cm}$. deep by $500 \mathrm{~cm}$. wide. The subsurface is divided into two sets of small divisions (rectangular blocks in different sizes). Each division is called an element in FEM. The center region ( 50 by 90 $\mathrm{cm}$.) is the region of interest and consists of 45 equal-size rectangular elements. The gray shaded region located outside the center region is the region of influence that consists of 99 unequal-size rectangular elements. The 3-D model of the experimental site for computing the potentials was an extension of 2-D. The 3-D model consisted of seven cross sections of 2-D; the center five cross sections had 10-cm thickness; the two side cross sections had 150$\mathrm{cm}$ thickness; and only the center cross section had current

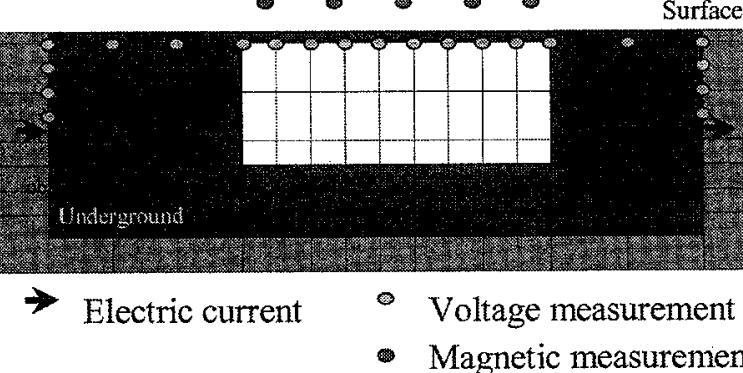

Figure 3: Finite Element Model for Field Demonstrations induced. The center region of 3-D was blocked into 245 unequal-sized cubics ( 5 by 7 by 5 ).

Steps 4 follow the SPSA algorithm. Note that ECOL follows two distinct parametric approaches, one (defined by [2]) for estimating the conductivity of the elements in the 50 - by 90 -cm middle section for 2 -D model (50- by 120 - by $50-\mathrm{cm}$ middle section for 3-D), and the other for the elements in the influence region outside the middle section. The conductivity values of the elements in the latter section are not optimized; they are kept at the same values throughout the iterations. The loss function mentioned in the SPSA algorithms is the sum of the square differences of the measured potential differences versus the FEM computed differences. The gain sequence used in SPSA is modified to avoid multiple solutions. The gain sequence \{c\} defined in [1] was kept as stepwise reduction (the value changed every 30 iterations). Discussion of the global optimization algorithm can be found in [3].

\section{Field Demonstrations}

Two field tests were conducted using magnetic field measurements. Test 1 used contact method to induce the current (as shown on Fig. 3). It is the same method used in Chin, et al. [9]. Chin, et al. [9] measured the differentialpotential at the dots beneath the surface as shown in Fig. 3; Test 2 took the magnetic field readings at 7 " above the surface in the area of interest as shown in Fig. 3. A total of 56 ( 7 rows by 9 columns) magnetic field readings were taken. As mention before, magnetic field measurement needs a detailed modeling technique. Therefore, the subsurface model was expanded to 3-D and the raw field measurements had been adjusted the effects from the induced in/out currents. Fig. 4 shows the simplified 3-D model (certain elements located at the outside influence region are omitted) at the upper-left corner and the reconstruction results on the right and bottom figure for the test. The single layers at right and bottom of the 3-D model represent the 

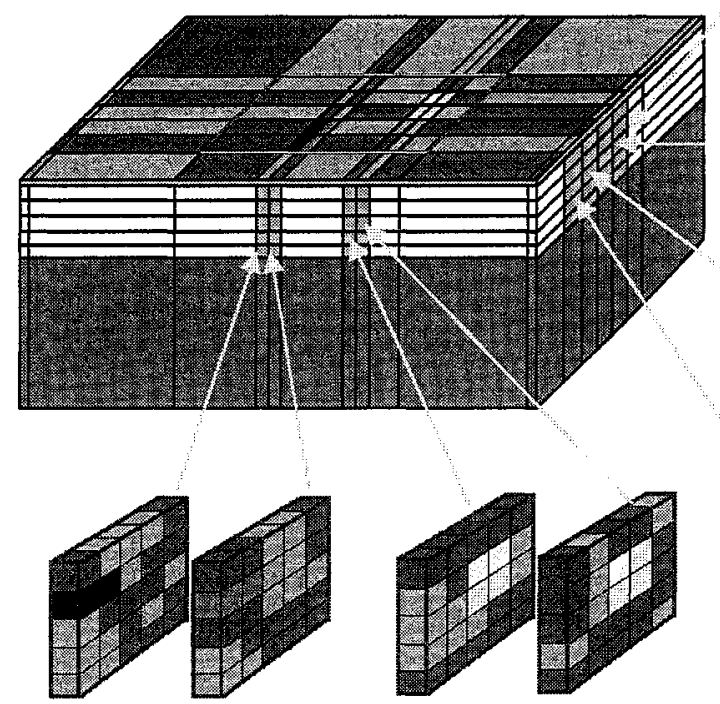

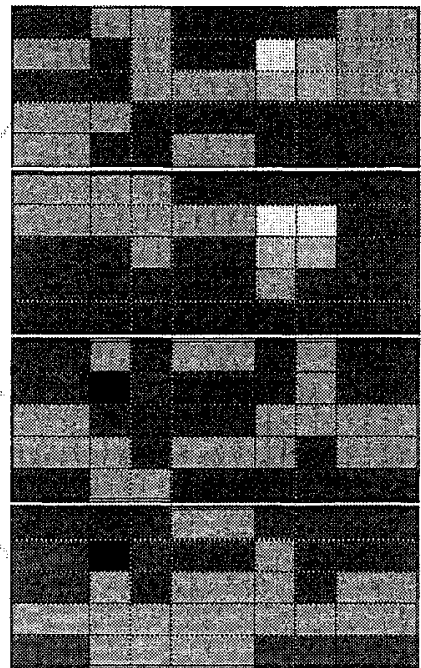

\begin{tabular}{lllllllll}
-4.04 & -3.95 & -3.94 & -4.04 & -4.05 & -4.00 & -3.93 \\
\hline
\end{tabular} $\begin{array}{llllllll}-3.99 & -4.00 & -3.94 & -4.01 & -3.6 & -2.1 & -3.97\end{array}$

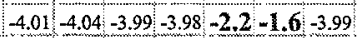
\begin{tabular}{llllllllll}
-3.95 & -3.98 & -4.04 & -4.03 & -4.10 & -4.02 & -4.04 \\
\hline
\end{tabular} $\begin{array}{llllllll}-3.97 & -4.03 & -4.07 & -3.96 & -4.01 & -4.04 & -4.01\end{array}$ \begin{tabular}{lllllllllll}
-3.97 & -3.98 & -3.98 & -4.03 & -4.03 & -4.05 & -4.07 \\
\hline
\end{tabular} $\begin{array}{llllllllll}-3.98 & -3.98 & -3.93 & -3.99 & -3.7 & -3.8 & -4.04\end{array}$ $\begin{array}{lllllllll}-4.07 & -4.00 & -3.97 & -4.01 & -\mathbf{3 . 1} & -\mathbf{2 . 9} & -4.06\end{array}$ \begin{tabular}{lllllllll}
-4.02 & -4.09 & -4.03 & -4.00 & -3.99 & -4.03 & -4.00 \\
\hline
\end{tabular} $\begin{array}{lllllllll}-4.05 & -4.00 & -4.02 & -4.00 & -4.03 & -4.04 & -4.01\end{array}$ \begin{tabular}{llllllllll}
-4.01 & -3.99 & -4.07 & -3.98 & -4.01 & -3.97 & -4.03 \\
\hline
\end{tabular} $\begin{array}{llllllll}-4.05 & \mathbf{4} & \mathbf{9} & -\mathbf{4 . 1} & -4.02 & -4.03 & -3.99 & -4.06\end{array}$

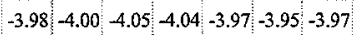
$\begin{array}{lllllllll}-3.99 & -3.94 & -4.03 & -3.96 & -3.90 & -4.03 & -3.95\end{array}$ $\begin{array}{lllllll}4.09 & -3.99 & -3.93 & -4.01 & -4.01 & 4.04-4.02\end{array}$ $-4.00-4.02-4.01+3.98-4.05-4.04-4.03$ $\mathbf{- 4 . 1}-\mathbf{5 . 1}-\mathbf{- 4 . 1}-4.08-3.93-4.02+4.07$ \begin{tabular}{lllllllllll}
-4.01 & -3.99 & -4.02 & -3.98 & -3.95 & -4.00 & -3.95 \\
\hline
\end{tabular} \begin{tabular}{ll|l|l|l|l|l|l|l|l|l|l|l|l}
-3.99 & -3.99 & -3.92 & -3.97 & -3.95 & -3.98 & -3.97 \\
\hline
\end{tabular} \begin{tabular}{lllllll}
-4.02 & -3.96 & -3.95 & -3.99 & -4.02 & -4.01 & -4.06 \\
\hline
\end{tabular}

$\square$ Elements contain metal material

Elements contain plastic material 질 Elements contain regular soil

Figure 4: Reconstruction of the Conductivities for Test 1 (The table entries are in log scale)

cross-sections of the relevant parts of the subsurface model where the arrows are pointing. The shades on the blocks on the top of the 3-D model are the indicators of where the objects could be inside of the subsurface. The different colors and shades on the cross-sections represent the electrical conductivities of the elements. The blue (darker) shades represent lower conductivity elements; the yellow (lighter) shades represent higher conductivity elements; the green shades represents elements with the conductivity in the soil level. The numbers on the tables are the log conductivity values of the elements. The objects may occupy part of elements; therefore, the lower and the higher highlighted numbers showed on the table are smaller or larger than the log conductivity values for the pure metal or plastic. The highlighted locations and their conductivity values are matched to the actual object locations and the conductivity level. The estimated locations and the conductivity values agreed with the actual buried objects with a small marginal error.

Test 2 consists of two cases, both of which used the non-contact method as shown in Fig. 1, the top view of the test site. Case 1 was conducted with three buried objects, two plastics and one metal, as indicated in Fig. 1. Case 2 was conducted shortly after Case 1 with all objects removed and holes partially refilled as indicated on the table in Fig 5, the row entries indicated as "Cond. Estimates (objects were removed)". The actual locations of the buried objects are listed on the table in Fig. 5, the row entries with label "Actual Loc."; the origin of the axes is located at the center of interested area on the surface. The unit of the locations is in feet. The number of magnetic field readings and FEM modeling structure are exactly the same as in Test 1. Fig. 5 shows the test results for both cases. The estimated and the actual locations for the buried objects are plotted on the top figure in Fig. 5 and listed as a row entry indicated with "Loc. Estimates" and "Actual Loc.". The row entries labeled with "Cond. Estimates" are the estimated conductivity from both cases. The conductivity estimates for Case 2 has a description, "(Obj. were Removed)". The estimates of Case 1 show that ECOL estimated the object locations within a radius of 1.2-ft from the actual locations and the conductivities are in expected range for both plastic and metal materials. Only conductivity values were estimated in Case 2 at the locations found the objects in Case 1. The estimated conductivity values in Case 2 are also in expectation, the

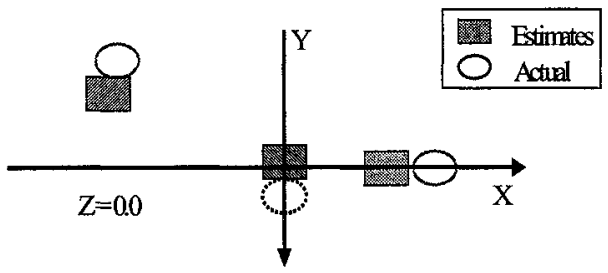

Center Loeations (X, Y, \&Zinft) and Conductivities

\begin{tabular}{|l|c|c|c|}
\hline Object Types & Plastic1 & Metal & Plastic 2 \\
\hline Loc. Fstimates & $-\mathbf{3 . 3},-0.8,-0.3$ & $0.2-0.1,-0.3$ & $1.8,-0.0,-0.3$ \\
\hline Actual Loc & $\mathbf{- 3 . 0 , - 1 . 5 - 0 . 2}$ & $0.0,0.5,-0.3$ & $3.0,-0.0,-0.2$ \\
\hline Cond Estimates & $\approx 1.0 \mathrm{E} 8$ & $\approx 1.0 \mathrm{E}+4$ & $\approx 1.0 \mathrm{E}-8$ \\
\hline $\begin{array}{l}\text { Cond Estimates } \\
\text { (Obj. nere Removed) }\end{array}$ & $\begin{array}{c}\approx 1.0 \mathrm{E}-9 \\
\text { (Opened hole) }\end{array}$ & $\begin{array}{c}\approx 1.0 \mathrm{E}-4 \\
\text { (Filledw.Soil) }\end{array}$ & $\begin{array}{c}\approx 1.0 \mathrm{E}-6 \\
\text { (Partially Filled) }\end{array}$ \\
\hline
\end{tabular}

Figure 5: Test Results for Field-test 2 
hole filled with original soil was estimated with soil conductivity, the open hole was estimated with near the air conductivity, and the partially filled hole with the conductivity between soil and air values.

Note that these experiments were conducted under a virtual blindfold condition, in which the algorithm made no a priori assumption about either the character of the objects or the conductivity of the soil.

\section{Conclusions}

The purpose of the Electrical Conductivity Object Locator is to generate an internal map of the location, size, and conductivity of all objects in a suspected site having plastic and/or metal mines. In this work, we have demonstrated that the ECOL technique is able to locate small-sized plastic and metal objects buried in shallow depths in cluttered soil.

The ECOL technique assumes spatial nonuniformity for conductivity of the soil subsurface. It divides the subsurface space into several elements and assumes that the objects of interest are present within some of those elements. The technique injects a small-amplitude, low frequency electrical current into the soil and measures the resulting electrical potentials at the soil surface. The conductivity of each element of the subsurface is reconstructed using the injected current as the input parameter and the measured potentials as the boundary condition. A sequence of algorithms, all of which were developed at JHU/APL, is used in the reconstruction procedure. The heart of the procedure is the Simultaneous Perturbation Stochastic Approximation (SPSA). Unlike conventional gradient techniques, SPSA can and does reconstruct conductivity maps successfully when accurate gradient (potential) data are available, and this is possible even when the gradient data are inaccurate or contaminated with noise. Under most field conditions, one should expect and be prepared to deal with measurement inaccuracies, as well as noise in the data. The success of the SPSA algorithm is attributed to its ability to reconstruct even under extreme conditions of noise and inaccuracies in the input parameters and boundary conditions.

Another practical advantage of the ECOL technique is that the current can be injected into the soil from a location that is away from the area of interest or where mines are presumed present. Both contact and non-contact methods are demonstrated. The contact method uses differential potentials (Chin, et al. [9]) and magnetic field measurement in 2-D and 3-D sitting. The non-contact method uses magnetic field measurement with 3-D sitting that is very sensitive to the input, and takes longer time to process.

\section{Acknowledgements}

The Johns Hopkins University Applied Physic Laboratory IR\&D funds solely supported this work.

\section{References}

[1] Spall, J. C., 1988, “A Stochastic Approximation Algorithm for Large-Dimensional System in the Kiefer-Wolfowitz Sitting," Proceedings of IEEE Conference in Decision Control, pp. 1544-1548.

[2] Spall, J. C., 1992, "Multivariate Stochastic Approximation Using a Simultaneous Perturbation Gradient Approximation," IEEE Transactions On Automatic Control, Vol. 37, pp. 332-341.

[3] Chin, D. C., 1994, "A More Efficient Global Optimization Algorithm Based on Styblinski and Tong," Neural Networks, Vol. 7, No. 2, pp. 573-574.

[4] Brown, B. H., D. C. Barber, W. Wang, Liquin Lu, A. D. Leathard, R. H. Smallwood, A. R. Hampshire, R. Mackay, and K. Hatzigalanis, 1994, "Multi-Frequency Imaging and Modeling of Respiratory Related Electrical Impedance Changes," Physiol. Meas., Vol. 15, A1-A12.

[5] Brown, B. H., D. C. Barber, A. H. Morice, and A. D. Leathard, 1994, "Multi-Frequency Imaging and Modeling of Respiratory Related Electrical Impedance Changes," IEEE Trans. Biomed. Eng., Vol. 41, No. 8, pp. 729-733.

[6] Smith, R. W., I. L. Freeston, and B. H. Brown, 1995, "A Real-Time Electrical Impedance Tomography System for Clinical Use- Design and Preliminary Results," IEEE Trans. Biomed. Eng., Vol. 42, No. 2, pp. 133-140, and references therein.

[7] Wexler, A., B. Fry, and M. R. Neuman, 1985, "Impedance-Computed Tomography Algorithm and System," Applied Optics, Vol. 24, No. 23, pp. 39853993.

[8] Cook, R. D., G. I. Saulnier, D. G. Gisser, J. C. Goble, J. C. Newell, and D. Isaacson, 1994, "ACT3: A HighSpeed, High-Precision Electrical Impedance Tomograph," IEEE Trans. Biomed. Eng., Vol. 41, No. 8, pp. 713-722, and references therein.

[9] Chin, D. C., R. Srinivasan, and R. Ball, 1999, "Discrimination of Buried Plastic and Metal Objects in Subsurface Soil," Information Processing for Remote Sensing, Ed. Chen CH, World Scientific, New Jersey, pp. $565-570$.

[10] K. Bathe (Ed.), Finite Element Procedures in Engineering Analysis, The Southeast Book Company, Prentice-Hall, Inc., New Jersey, 1982.

[11] P. Hammond (Ed.), Electromagnetism for Engineers, The Macmillan Company, Pergamon Press, Limited, New York, 1964. 